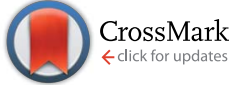

Cite this: J. Mater. Chem. A, 2016, 4, 17801

Received 20th September 2016 Accepted 20th October 2016

DOI: 10.1039/c6ta08155j

www.rsc.org/MaterialsA

\section{Plasma surface functionalization induces nanostructuring and nitrogen-doping in carbon cloth with enhanced energy storage performance $\uparrow$}

\author{
Bo Ouyang, $\star^{a}$ Yongqi Zhang, $\$^{b}$ Ying Wang, ${ }^{\text {ac }}$ Zheng Zhang, ${ }^{d}$ Hong Jin Fan*b \\ and Rajdeep Singh Rawat*a
}

A facile, one-step and environmentally-friendly strategy for the preparation of hierarchical nitrogen-doped carbon cloth ( $\mathrm{hNCC}$ ) is presented via nitrogen plasma processing of commercial carbon cloth. In addition to $\mathrm{N}$-doping, the RF plasma treatment induces nanostructuring, thus significantly increasing the surface area and liquid electrolyte wettability. The untreated carbon cloth (CC) has negligible Li-ion and supercapacitive storage capacities. However, after plasma treatment, the obtained hNCC delivers dramatically enlarged capacities. Specifically, the enhancement is by three times for Li-ion storage (150 $\mathrm{mA} \mathrm{h} \mathrm{g}^{-1}$ versus $50 \mathrm{~mA} \mathrm{~h} \mathrm{~g}^{-1}$ at $100 \mathrm{~mA} \mathrm{~g}^{-1}$ ), and by three orders of magnitude for pseudocapacitance ( $391 \mathrm{mF} \mathrm{cm}{ }^{-2}$ versus $0.12 \mathrm{mF} \mathrm{cm}^{-2}$ at $4 \mathrm{~mA} \mathrm{~cm}^{-2}$ ). The effect of power-dependent plasma treatment for optimized performance is also investigated. We propose a plausible mechanism for achieving a hNCC architecture with highly enhanced ion/charge storage properties. Our research provides an approach to fabricate $\mathrm{N}$-doped carbon materials with a controllable surface morphology and electrochemical properties. This deterministic and plasma-based method of preparing hNCC may offer new opportunities in the design and fabrication of high-performance carbon-based electrodes for energy storage devices.

\section{Introduction}

High performance electrochemical energy storage devices such as supercapacitors (SCs) and lithium ion batteries (LIBs) have received considerable research interest as a result of there being limited fossil-fuel reserves and ever-increasing demands for renewable energy. ${ }^{1}$ Among the various electrode materials, carbon based materials, such as carbon spheres, ${ }^{2}$ carbon nanotubes ${ }^{3}$ and graphenes ${ }^{4}$ have been widely applied as anodes for energy storage devices because of their high surface area, excellent electrical conductivity, and extraordinary electrochemical inertness. However, these carbon based materials suffer from low specific capacitance due to their intrinsic electric double-layer mechanism, limiting the energy density of supercapacitors. ${ }^{5}$ Given their electrochemical properties and

${ }^{a}$ Natural Sciences and Science Education, National Institute of Education, Nanyang Technological University, 637616, Singapore. E-mail: rajdeep.rawat@nie.edu.sg

${ }^{b}$ School of Physical and Mathematical Sciences, Nanyang Technological University, Singapore 637371, Singapore.E-mail:fanhj@ntu.edu.sg

${ }^{c}$ School of Materials Science and Engineering, Nanyang Technological University, Singapore 639798, Singapore

${ }^{d}$ Institute of Materials Research and Engineering, A*STAR (Agency for Science, Technology and Research), \#08-03, Fusionopolis Way, Innovis, 138634, Singapore

† Electronic supplementary information (ESI) available. See DOI: 10.1039/c6ta08155j

\$ These authors contributed equally to this work. fabrication cost, it is highly desirable to develop a facile, scalable and controllable approach to boost the capacitive performance of carbon-based electrodes.

Compared to elaborate and expensive carbonaceous architectures (e.g. CNTs and graphene), commercial carbon cloth (CC), composed of numerous uniform carbon microfibers, is an inexpensive and highly conductive textile with excellent mechanical flexibility and strength. ${ }^{6}$ However, due to its poor electrochemical properties, small specific surface area and poor porosity, CC is not useful for direct utilization as an electrode material. However, CC holds great promise as the three dimensional (3D) support substrate and collector in the fabrication of flexible electrodes. Various electrochemically active materials, such as $\mathrm{MnO}_{2},{ }^{7} \mathrm{Co}_{3} \mathrm{O}_{4},{ }^{8} \mathrm{TiO}_{2}$ (ref. 9) and $\mathrm{ZnO}^{10}$ have been anchored on the surface of CC for enhanced electrochemical performance along with remarkable flexibility. Nonetheless, due to the potential incompatibility issue between electroactive materials and the $3 \mathrm{D}$ conductive scaffold, the direct anchoring of electroactive materials with an optimal porous structure on such a $3 \mathrm{D}$ porous substrate still remains a considerable challenge. To look beyond 3D electrode architectures and to upgrade this technology towards high performance energy storage devices, major consideration is given to the surface activation of the current collector. Recently, Wang et al. demonstrated a chemical exfoliation strategy to enhance the specific capacitance of CC (to $88 \mathrm{mF} \mathrm{cm}^{-2}$ ). ${ }^{\mathbf{1 1}}$ Even though 
such an approach led to large amounts of oxygen-containing functional groups and a considerably enhanced specific surface area of CC during chemical activation, the introduced $\mathrm{C}-\mathrm{O}$ bonding may still lower the conductivity of the entire electrode and thus limits the eventual increase in specific capacitance.

Remarkably, artificial doping with heteroatoms, particularly nitrogen atoms, can effectively improve the electronic conductivity, electron-donor tendency and surface wettability of carbon materials, thus indicating that doped carbon materials possess an excellent ability to exhibit ion/charge storage properties. ${ }^{\mathbf{1 2 - 1 4}}$ Recently, significant progress has been made in developing high performance carbon anodes by combining the advantages of carbon nanostructures and heteroatom doped carbon materials, such as $\mathrm{N}$-doped carbon nanocages, ${ }^{13} \mathrm{~N}$-doped carbon foams ${ }^{15}$ and $\mathrm{N}$-doped porous graphene. ${ }^{14}$ It has also been reported that after nitrogen doping, pseudo-capacitance can be generated due to the interaction between the electrolyte and the $\mathrm{N}$ species on the surface, resulting in the enhancement of capacitive properties. ${ }^{\mathbf{1 6}}$

In this work, we report a novel one-step nitrogen plasma processing strategy to simultaneously achieve a hierarchical 3D nanostructured network along with the introduction of nitrogen-containing functional groups onto the commercial CC surface, resulting in distinctly enhanced wettability and electrochemical performance. The hierarchical nitrogen doped carbon cloth (hNCC) electrode exhibited a three-fold increase in specific capacity to $150 \mathrm{~mA} \mathrm{~h} \mathrm{~g}{ }^{-1}$ at a current density of $100 \mathrm{~mA} \mathrm{~g}^{-1}$, compared to $50 \mathrm{~mA} \mathrm{~h} \mathrm{~g}{ }^{-1}$ for untreated CC at the same current density. In addition, the hNCC electrode also turned out to be an excellent electrode for supercapacitors with a specific capacitance of $391 \mathrm{mF} \mathrm{cm}^{-2}$ at a current density of $4 \mathrm{~mA} \mathrm{~cm}^{-2}$ and outstanding long-term cyclic durability (without any significant decay in capacitance after 10000 cycles). Compared with the previous strategies for activating carbon materials, the approach presented in this paper is relatively simple, effective and environmentally friendly. Moreover, optical emission spectroscopy of nitrogen plasma was conducted to deduce a plausible mechanism of hNCC formation, to formulate a generic approach for controllable nitrogen activation of the plasma treated CC.

\section{Experimental}

\subsection{Preparation of hNCC}

The hNCC sample was prepared using our RF-plasma system as shown in Fig. S1. $\dagger$ In a typical procedure, commercial CC $(1 \times$ $1.5 \mathrm{~cm}^{2}$ ) was washed with ethanol, acetone, and deionized water, in turn, and subsequently dried in a vacuum oven. The as-obtained CC was then placed inside the quartz tube reactor of the RF-plasma system between the two capacitively coupled $\mathrm{RF}$ electrodes at a distance of $\sim 5 \mathrm{~cm}$ from the left electrode. Afterwards, the tube was evacuated using a combination of the rotary and turbo pumps. The nitrogen was made to flow into the system at a flow rate of $15 \mathrm{sccm}$ and the pumping rates were adjusted using mechanical valves to keep the quartz tube reactor chamber pressure at $\sim 0.2$ mbar. Nitrogen plasma was created using $500 \mathrm{~W}$ RF power from a $13.56 \mathrm{MHz}$ Caeser136 RF generator, coupled through a Navio Matching network to the RF electrodes. The $500 \mathrm{~W}$ RF plasma treatment of the CC was done for $15 \mathrm{~min}$ (15 $\mathrm{min}$ @ $500 \mathrm{~W})$.

\subsection{Characterization}

Field-emission scanning electron microscopy (FESEM) analysis was carried out using a JEOL JSM-6700F scanning electron microscope at an accelerating voltage of $10 \mathrm{kV}$. Transmission electron microscopy (TEM) measurements were performed using a JEOL-2010 UHR high resolution transmission electron microscope at an accelerating voltage of $200 \mathrm{kV}$. X-ray diffraction (XRD) patterns were collected using a D8 advanced diffractometer equipped with $\mathrm{Cu} \mathrm{K} \alpha$ radiation $(\lambda=0.15406 \mathrm{~nm})$. $\mathrm{X}$-ray diffraction spectroscopy (XPS) analysis was conducted using a Thermal Scientific Theta Probe system XPS with a monochromatic $\mathrm{Al} \mathrm{K} \alpha(486.7 \mathrm{eV}) \mathrm{X}$-ray source. The binding energy of the $\mathrm{C} 1 \mathrm{~s}$ peak from $\mathrm{sp}^{2}$-bonded carbon at $284.5 \mathrm{eV}$ was used as a reference for correction. Raman spectra were obtained using a WITEC CRM200 Raman system with a 488 $\mathrm{nm}$ excitation laser. The optical emission spectroscopy (OES) of nitrogen plasma was conducted using an Ocean Optics HR4000 spectrometer.

To measure the electrochemical performance of all the synthesized materials, coin-type cells (CR 2016) were assembled in an argon-filled glove box with both moisture and oxygen content below $0.1 \mathrm{ppm}$. The coin cell was assembled using a metallic lithium foil as the counter-electrode, $1 \mathrm{M}$ $\mathrm{LiPF}_{6}$ in ethylene carbonate (EC)-dimethyl carbonate (DME) (1: 1 in volume) as the electrolyte, and a polypropylene (PP) film (Celgard 2400) as the separator. Pristine and nitrogen plasma treated CCs were directly used as the working electrode in the coin cell without the addition of any conductive or binding materials. The galvanostatic charge/discharge tests were performed using a NEWARE battery testing system at different current rates. Cyclic voltammetry (CV) measurements were conducted on a $\mathrm{CHI} 760 \mathrm{D}$ electrochemical workstation using a potential range of $0.05-1.5 \mathrm{~V}\left(v s . \mathrm{Li} / \mathrm{Li}^{+}\right)$. The electrochemical impedance spectroscopy (EIS) was performed using a CHI 760D electrochemical workstation in the frequency range of $1 \times 10^{5}$ to $0.01 \mathrm{~Hz}$. The supercapacitive properties of the electrodes were measured in a $1 \mathrm{M} \mathrm{KOH}$ electrolyte using a three electrode cell configuration (CHI 6604D).

\subsection{Calculation}

The areal capacitance $\left(C_{\mathrm{a}}, \mathrm{F} \mathrm{\textrm {cm } ^ { - 2 }}\right)$ of the electrode was calculated from its $\mathrm{CV}$ curves using the following equation:

$$
C_{\mathrm{a}}=\frac{Q}{S \Delta U}=\frac{\oint I \mathrm{~d} U}{2 S v \Delta U},
$$

where $Q$ is the average charge, $\Delta U$ is the potential window, $v$ is the scan rate, and $S$ is the active surface area of the working electrode and $I$ is the current density. There is a factor 2 because one $\mathrm{CV}$ cycle includes the charge and discharge processes. The integral contains the charge of these two processes. 


\section{Results and discussion}

\subsection{Plasma functionalization and characterization}

The FESEM images of the untreated and $15 \mathrm{~min}$ (a) $500 \mathrm{~W}$ nitrogen RF-plasma treated CCs are shown in Fig. 1a-d and S2a. $\uparrow$ From Fig. 1d and S2a, $\dagger$ it can be seen that the untreated CC was composed of numerous interlaced carbon fibers with a smooth surface. After nitrogen RF-plasma treatment, the surface of the carbon fibers became extremely rough (Fig. 1b and c), indicating the successful modification of the carbon fiber surface by plasma treatment. The inset in Fig. 1c clearly shows the formation of narrow-size distributed uniform nanoparticle decorated CC, with the particle size in the range of $35 \pm$ $5 \mathrm{~nm}$, on the nitrogen plasma treated CC. Notably, the method is suitable for large area treatment of CC with nearly uniform nano-structurization of an area of about $1.5 \mathrm{~cm}^{2}\left(1 \times 1.5 \mathrm{~cm}^{2}\right.$, Fig. S2b $\dagger$ ). Moreover, the original excellent flexibility and mechanical strength of the CC was still preserved after the nitrogen plasma treatment (Fig. S2c $\dagger$ ). The elemental distribution of the hNCC was further characterized by energy dispersive spectroscopic (EDS) mapping (Fig. S2d $\dagger$ ). It can be observed that the $\mathrm{N}$ elements are uniformly dispersed onto the surface of the hNCC sample. Fig. 1c shows the low-magnification TEM image, which clearly reveals the formation of 20-40 $\mathrm{nm}$ thick spherical nanoparticles on the hNCC, confirming the SEM imaging results. High resolution TEM (HRTEM, insert of Fig. 1c) shows one of the spherical nanoparticles with a graphite-like structure with an inter-planar spacing of $\sim 0.34 \mathrm{~nm}$, indicating graphitic phase formation on the hNCC surface due to nitrogen plasma
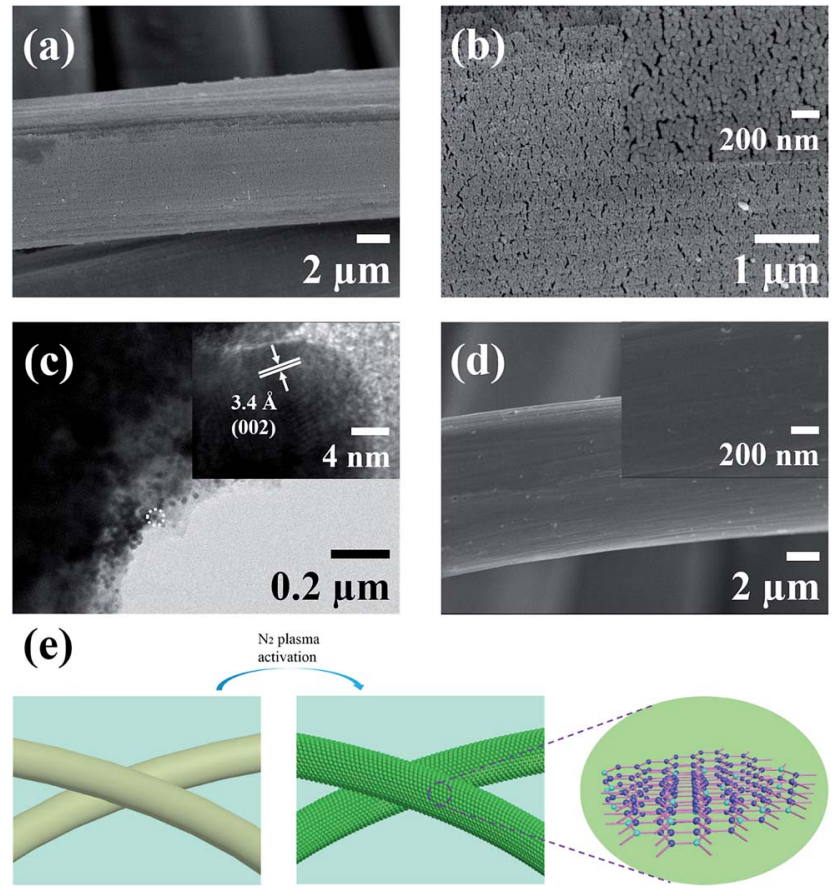

Fig. 1 The morphology of the hNCC sample. (a) and (b) FESEM images and (c) TEM and HRTEM (inset) images; (d) FESEM image of the corresponding untreated $\mathrm{CC}$; (e) schematic of $\mathrm{hNCC}$ preparation by nitrogen plasma activation. treatment. Fig. 1e shows the schematic of nanostructural decoration (observed through the SEM and TEM images) and nitrogen functionalization (discussed in the next paragraph) of the hNCC sample.

To confirm the functionalization and surface modification of the nitrogen plasma treated CC surface, Raman spectroscopy, $\mathrm{X}$-ray diffraction (XRD) and X-ray photoelectron spectroscopy (XPS) were performed. The Raman spectra of the hNCC and untreated CC samples, shown in Fig. 2a, reveal a pronounced D band (a disordered band) at $\sim 1350 \mathrm{~cm}^{-1}$ and a $\mathrm{G}$ band (associated with in-plane vibration of the graphite lattice) at $\sim 1590 \mathrm{~cm}^{-1} .{ }^{17}$ The $\mathrm{D}$ to $\mathrm{G}$ peak intensity ratio of the hNCC sample is estimated to be 1.61 which is much larger than that of 0.93 for the untreated CC, indicating greater disorder/defects in the hNCC sample. The greater disorder in the hNCC sample can be attributed to the presence of nitrogen containing functional groups on the surface of the hNCC sample together with dense reactive graphitic edges. ${ }^{18}$ Additionally, a small upshift in the D and $\mathrm{G}$ band positions for the hNCC sample (Fig. S3 $\dagger$ ) indicates that the hNCC nanostructure has a greatly enhanced defect density. ${ }^{2}$ Fig. 2 b shows the X-ray diffraction (XRD) spectra of the hNCC and untreated CC samples. The wider base of the (002) diffraction peak centered at $\sim 26^{\circ}$ indicates that both of these samples are composed of amorphous and graphitic types of carbon. ${ }^{19}$ The deconvolution of the wide peak centered at $\sim 26^{\circ}$ for the hNCC and untreated CC samples is shown in Fig. S4a and S4b. $\dagger$ The sharp deconvoluted peak centered at $\sim 26^{\circ}$ and the broad peak at $\sim 22^{\circ}$ are representative of the graphitic carbon and amorphous-like carbon contributions, respectively. The percentage of graphitic structure in the hNCC is enhanced to about $66 \%$, from about $60 \%$ in the untreated CC, which further confirms the results of HRTEM. The increased graphitic percentage in the hNCC sample can be attributed to energetic nitrogen plasma processing of the treated CC surface. High-resolution X-ray photoelectron spectroscopy (refer to
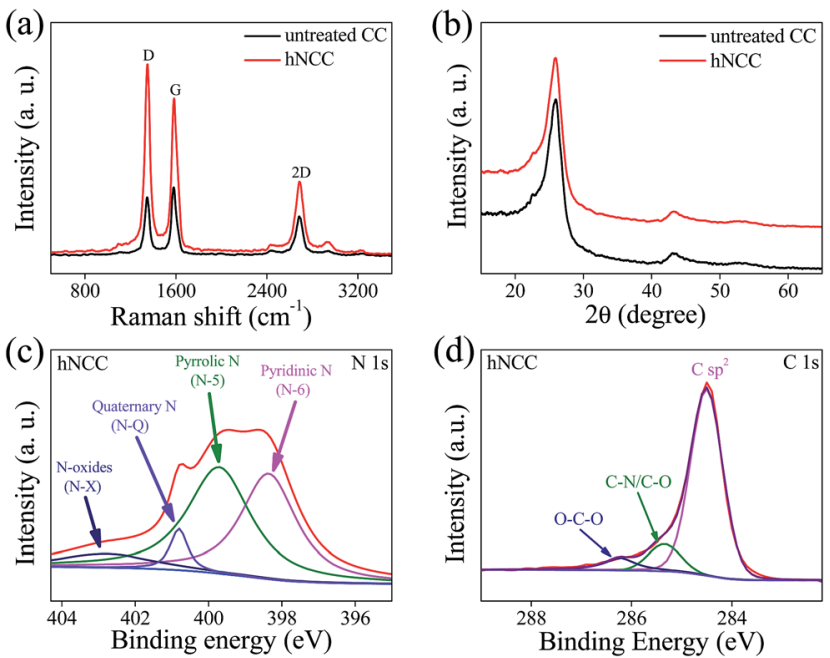

Fig. 2 (a) Raman spectra of the hNCC and untreated CC samples; (b) XRD pattern of the hNCC and untreated CC samples; (c) high resolution XPS spectra of N 1s in the hNCC sample; (d) high resolution XPS spectra of $\mathrm{C}$ 1s in the hNCC sample. 
Fig. 2c and S5a $\dagger$ ) confirms the existence of $\mathrm{N}$-containing species (5.6 atom\%) in the hNCC sample, which include pyridinic $\mathrm{N}$ $(\mathrm{N}-6,398.4 \mathrm{eV})$, pyrrolic $\mathrm{N}(\mathrm{N}-5,399.7 \mathrm{eV})$, quaternary $\mathrm{N}(\mathrm{N}-\mathrm{Q}$, $400.8 \mathrm{eV}$ ) and oxidized $\mathrm{N}(\mathrm{N}-\mathrm{X}, 402.7 \mathrm{eV})$ species, which can provide electrochemically active sites and good conductivity with enhanced capacitive properties of the material. ${ }^{20}$ Notably, the percentage of $\mathrm{N}$ atoms on the edge of the graphite plane (N-5, N-6 and N-X) is 93.7\%, much higher than in the middle of the graphite plane (N-Q), implying the existence of nitrogen at the more effective active sites (refer to Table $\mathrm{S} 1 \dagger$ ). ${ }^{21}$ No nitrogen species were detected in the untreated CC sample. The nitrogen doping in the hNCC sample is expected to enhance the surface polarity, electric conductivity, and electron-donor tendency of the carbon materials, which can provide not only enhanced capacity for lithium ion batteries but also additional pseudocapacitance, and improved rate performance and cycling stability for supercapacitor applications. ${ }^{22}$ Owing to its nanostructured, N-doped and conductive architecture with a 3D hierarchical inherent network, it is expected that the hNCC should have significantly improved electrochemical performance for lithium ion batteries and supercapacitors. Moreover, as expected, the C 1s XPS peak (Fig. 2d) can be deconvoluted into a dominant component of $\mathrm{sp}^{2}$ carbon at $284.5 \mathrm{eV}$, along with two weaker bands associated with $\mathrm{C}-\mathrm{O}$ and $\mathrm{C}-\mathrm{N}$ at $285.4 \mathrm{eV}$ and $\mathrm{O}-\mathrm{C}-\mathrm{O}$ at $286.2 \mathrm{eV}$, which also demonstrates the nitrogen doping on the CC surface. In comparison, the peak at $285.4 \mathrm{eV}$ in untreated CC (Fig. S5b ${ }^{\dagger}$ ) exhibits much weaker intensity, indicating that a relatively smaller amount of the functional group is attached to its surface.

The surface wettability of the electrode material is important for its application in electrochemical devices. To investigate the effect of $\mathrm{N}$-doping on the wettability, water contact angle measurements were conducted for both the hNCC and the untreated CC (data shown in Fig. S6 $\dagger$ ). The untreated CC is found to be very hydrophobic with a contact angle of about $141.6^{\circ}$. In contrast, the plasma activated hNCC becomes very hydrophilic with the contact angle reduced to $21.3^{\circ}$ after resting the water droplet on the surface for $5 \mathrm{~s}$. The XPS result shown in Fig. 2d confirms the $\mathrm{C}-\mathrm{N}$ and $\mathrm{C}-\mathrm{O}$ bonds on the plasma processed hNCC sample surface. While a small amount of $\mathrm{C}-\mathrm{O}$ bonds existed on the untreated carbon cloth (refer Fig. S5 $\dagger$ ), the $\mathrm{C}-\mathrm{N}$ bonds were formed only after $\mathrm{N}$ plasma treatment. The $\mathrm{C}-\mathrm{N}$ bonding is polar due to the higher electronegativity of $\mathrm{N}$ (3.04) compared to that of $\mathrm{C}$ (2.55), making it hydrophilic towards polar water molecules. Other research groups have also attributed the enhanced hydrophilic property of nitrogen doped carbon materials to $\mathrm{C}-\mathrm{N}$ bond formation. ${ }^{23}$ Furthermore, the formation of a nano-structure on the surface of carbon materials can also impede the adsorption of water to some extent. ${ }^{24}$ Thus even though the hNCC surface is nanostructured, the much enhanced wettability of the hNCC is clearly due to the overriding effect of the polar $\mathrm{C}-\mathrm{N}$ bonds introduced via nitrogen doping.

\subsection{Enhancement in lithium ion storage properties}

The electrochemical properties of the hNCC electrode, together with the untreated CC electrode, were measured by configuring them as the laboratory-based CR2016 coin cells. The hNCC and untreated CC (as the anode) were investigated against Li metal under galvanostatic cycling conditions at room temperature in the voltage window of $0.005-3.0 \mathrm{~V}$. Fig. S7† shows the initial charge-discharge profiles for the hNCC and untreated CC samples at $100 \mathrm{~mA} \mathrm{~g}^{-1}$. The charge-discharge profiles are in accordance with the cyclic voltammetry (CV) analysis given in Fig. 3a, which shows the CV curves of the hNCC at a fixed scan rate of $0.1 \mathrm{mV} \mathrm{s}^{-1}$ in the voltage range of $0.005-1.5 \mathrm{~V}$. As can be seen in Fig. 3a, the first cathodic peak is centered at $\sim 0.8 \mathrm{~V}$, which corresponds to the formation of a solid electrolyte interphase (SEI) on the surface of the electrode. ${ }^{25}$ The CV curves overlap in the following cycles, indicating the formation of a stable SEI layer. The following cathodic peaks situated at $\sim 0.2 \mathrm{~V}$ are mainly attributed to lithium-ion insertion into the graphitic structure, which is an important indicator of lithium storage in a carbon-based architecture. In addition, the capacity above $0.5 \mathrm{~V}$ is attributed to the faradic capacitance on the surface or edge sites of graphitic carbon. To compare the relatively long-term cyclic performance of the hNCC and untreated CC, the cells were charged and discharged for 150 cycles at a current density of $100 \mathrm{~mA} \mathrm{~g}^{-1}$ between 0.005 and $3.0 \mathrm{~V}$. As shown in Fig. $3 \mathrm{~b}$, the hNCC electrode delivers a reversible capacity of about $140 \mathrm{~mA} \mathrm{~h} \mathrm{~g}^{-1}$ after 150 cycles, considerably higher than that of untreated CC $\left(50 \mathrm{~mA} \mathrm{~h} \mathrm{~g}^{-1}\right)$, with a corresponding coulombic efficiency of over $99 \%$. The rate capabilities for the untreated CC and hNCC based anodes were measured for 60 cycles from 100 to $1600 \mathrm{~mA} \mathrm{~g}^{-1}$ in the voltage range of 0.005 to $3 \mathrm{~V}$ (Fig. 3c). As can be seen, the hNCC anode exhibited the highest charge-discharge capacity of $159 \mathrm{~mA} \mathrm{~h} \mathrm{~g}^{-1}$ at $100 \mathrm{~mA} \mathrm{~g}^{-1}$, more than twice that of the untreated CC $\left(61 \mathrm{~mA} \mathrm{~h} \mathrm{~g}^{-1}\right)$. Additionally, the hNCC can sustain high capacities of 124, 85, 55 and $45 \mathrm{~mA} \mathrm{~h} \mathrm{~g}^{-1}$, with a coulombic efficiency of over $98 \%$, at current densities of 200, 400, 800 and $1600 \mathrm{~mA} \mathrm{~g}^{-1}$, respectively. These values are much higher than those of the untreated CC.
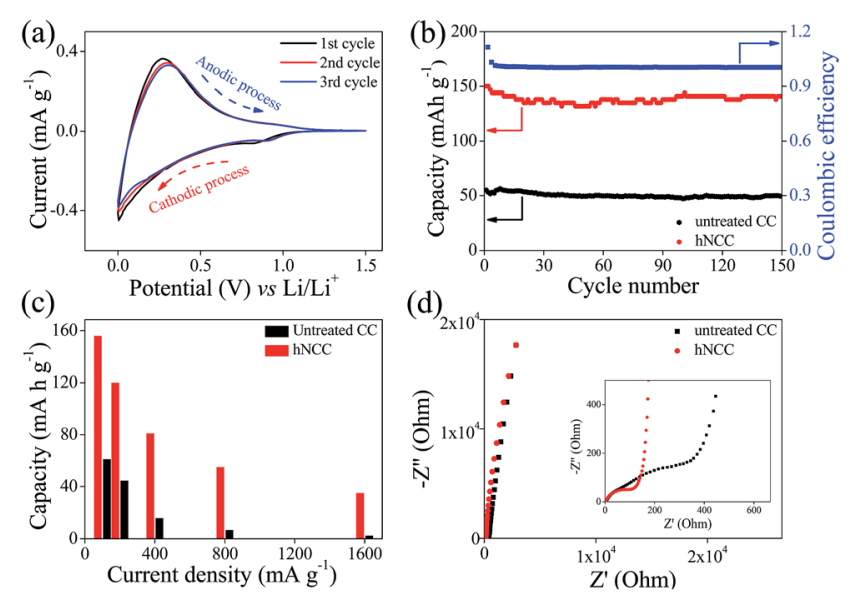

Fig. 3 The lithium ion storage properties of the hNCC compared with pristine CC. (a) CV curves of the hNCC sample; (b) cyclic stability of the hNCC and untreated CC electrodes at a current density of $100 \mathrm{~mA} \mathrm{~g}^{-1}$, and their coulombic efficiency; (c) rate performance of the hNCC and untreated CC electrodes at different current densities; (d) EIS spectroscopy of the hNCC and the untreated CC. 
Simultaneously, the enhanced conductivity of the hNCC electrode, demonstrated by electrochemical impedance spectroscopy (EIS) in Fig. 3d, could also be responsible for such a capacity enhancement in the frequency range of $1 \times 10^{5}$ to 0.01 Hz. Nyquist plots are shown in Fig. $3 d$. The results reveal that the hNCC electrode has a lower electrolyte resistance $\left(R_{\mathrm{S}}=2.9 \Omega\right)$ and charge transfer resistance $\left(R_{\mathrm{ct}}=133 \Omega\right)$ than the untreated CC electrode $\left(R_{\mathrm{S}}=4.2 \Omega\right.$ and $\left.R_{\mathrm{ct}}=172 \Omega\right)$. Compared to the untreated CC, the superior capacity of the hNCC is ascribed to the synergistic effects of nitrogen doping on the surface of CC, as well as the unique nano-structured surface of the hierarchical 3D architecture, which effectively facilitate the Li-ion transport property due to the decreased electrochemical resistance.

\subsection{Enhancement in supercapacitive properties}

Commercial untreated CCs have been widely employed as flexible and conductive substrates for metal oxide, nitride or carbide materials for supercapacitor applications. ${ }^{26}$ However, the untreated $\mathrm{CC}$ alone has a rather small and negligible electric double-layer capacitance. After the nanostructuring and $\mathrm{N}$ doping by plasma functionalization, we expect a significant increase in the supercapacitive charge storage function. The cyclic voltammetry (CV) curves of the untreated CC and hNCC electrodes at a scan rate of $200 \mathrm{mV} \mathrm{s}^{-1}$ are shown in Fig. 4a. The CV curve of the hNCC electrode exhibits a box-like shape in the potential range of 0 to $0.58 \mathrm{~V}$. The irregular shape of the $\mathrm{CV}$ curve for the hNCC sample suggests the presence of excess nitrogen containing functional groups on the outer surface that give rise to redox reactions. ${ }^{\mathbf{1 6}}$ However, importantly, the hNCC electrode exhibits a current density many orders of magnitude larger than that of the untreated CC electrode, indicating the remarkably enhanced charge storage capability of the hNCC
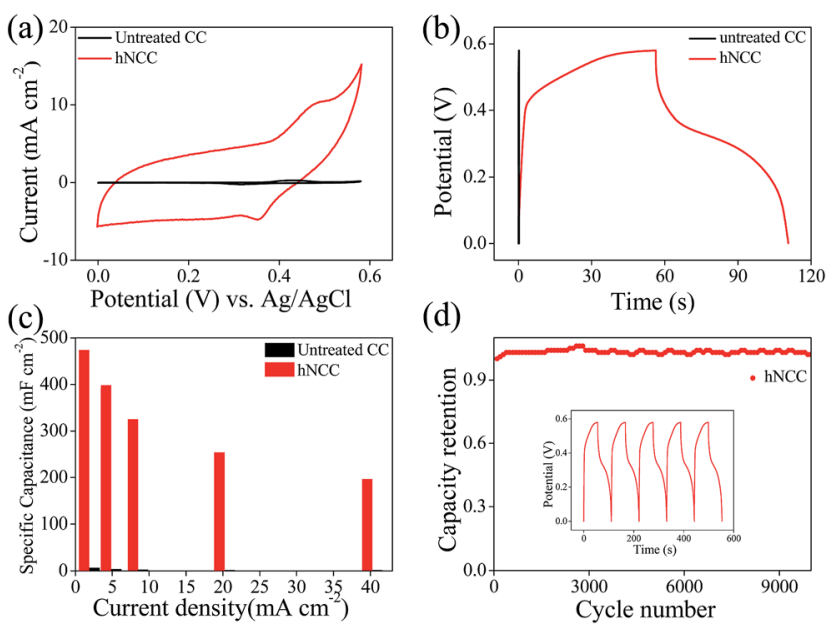

(d)

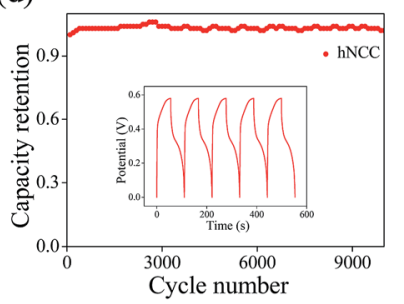

Fig. 4 The supercapacitive performance of the hNCC and untreated CC electrodes. (a) CV curves of the hNCC and untreated CC at $200 \mathrm{mV}$ $\mathrm{s}^{-1}$; (b) galvanostatic charge-discharge curves of the hNCC and untreated $C C$ electrodes at $4 \mathrm{~mA} \mathrm{~cm}^{-2}$; (c) specific capacitance as a function of the current densities of the hNCC and untreated CC electrodes; (d) cyclic stability of the hNCC electrode obtained at $4 \mathrm{~mA}$ $\mathrm{cm}^{-2}$ for 10000 cycles (the inset shows the last five cycles during the cyclic performance test). electrode. Fig. 4b shows the galvanostatic charge-discharge curves of the untreated CC and hNCC electrodes collected at a current density of $4 \mathrm{~mA} \mathrm{~cm}^{-2}$. The much larger discharge time of the hNCC again demonstrates its enhanced capacitive performance because of the contribution of pseudocapacitance (voltage plateau at $\sim 0.3 \mathrm{~V}$ ) by a large amount of nitrogen containing functional groups on the graphitic edges. Fig. 4c compares the specific capacitance of the untreated CC and hNCC electrodes at different current densities. Significantly, the hNCC electrode demonstrates a high specific capacitance of $391 \mathrm{mF} \mathrm{cm} \mathrm{cm}^{-2}$ at a current density of $4 \mathrm{~mA} \mathrm{~cm} \mathrm{~cm}^{-2}$, which is $\sim 3250$ times higher than that of the untreated CC electrode $\left(0.12 \mathrm{mF} \mathrm{cm}^{-2}\right)$. In addition, $\sim 39.8 \%$ of the specific capacitance was retained by the hNCC electrode as the current density increased from 2 to $40 \mathrm{~mA} \mathrm{~cm}{ }^{-2}$. Moreover, the hNCC electrode exhibited excellent cyclic stability without any decrease in the specific capacitance even after 10000 cycles, as shown in Fig. 4d. Additionally, the respective contribution of the pseudocapacitance and double-layer capacitance was also compared for the hNCC sample (related plots are provided in Fig. S $8 \dagger$ along with a detailed explanation). ${ }^{\mathbf{1 6 , 2 7}}$ The estimated pseudocapacitance contribution plays a critical role in the hNCC electrode, providing $72.5 \%$ of the total specific capacitance, while the double-layer capacitance contributes the rest (27.5\%). All these results conclusively confirm that the capacitive performance of the CC can be effectively increased by the singlestep nitrogen plasma activation method used in the present study (Table S2†).

Electrochemical impedance spectroscopy was conducted to investigate the electrochemical properties of the samples assembled for the supercapacitor configuration. As shown in Fig. $S 9, \dagger$ the absence of a semicircle in the high-frequency region indicates that the ionic conductivity at the electrode and electrolyte is extraordinary. Additionally, the calculated charge transfer resistances $\left(R_{\mathrm{ct}}\right)$ of the untreated CC and hNCC electrodes are $\sim 6.8$ and 1.7 , respectively. The considerably decreased $R_{\text {ct }}$ of the hNCC electrode reveals the greatly enhanced charge transfer properties. Additionally, a straight line with a slope of about $80^{\circ}$ is observed for both the samples in the low-frequency region, which corresponds to the semiinfinite Warburg impedance resulting from the frequency dependence of the ion diffusion/transport in the electrolyte, indicating the fast ion transport at the electrode-electrolyte interface. ${ }^{28}$ Moreover, the higher specific capacitance of the hNCC electrode can also be confirmed by the increased surface area. The surface areas of the hNCC and untreated CC samples were estimated by measuring the electrochemical capacitance of the film-electrolyte interface in the double-layer regime of the voltammograms. The specific capacitance $\left(C_{\mathrm{dl}}\right)$ was estimated to be $2.4 \mathrm{mF} \mathrm{cm}^{-2}$ for the hNCC and $0.23 \mathrm{mF} \mathrm{cm}^{-2}$ for the untreated CC (representative plots are provided in Fig S10†, together with a detailed explanation). According to the results, the ratio between the hNCC and untreated CC is estimated to be $10: 1$. Since the $C_{\mathrm{dl}}$ is proportional to the surface area of the materials, the hNCC electrode possesses a much higher active surface area than the untreated CC, thus it delivers both an enhanced specific surface area for double-layer capacitance and 
especially increased polar $\mathrm{CN}$ bonding networks for pseudocapacitance. Therefore, the significantly enhanced capacitance and cyclic durability of the hNCC electrode can be attributed: (1) to the incorporation of mainly nitrogen, and to some extent oxygen, functional groups onto the carbon fiber surface, and (2) to the increased surface area of the carbon fibers as a result of surface nanostructurization by nitrogen plasma treatment.

\subsection{Discussion on the activation mechanism}

In order to understand the nitrogen plasma activation on the surface of the commercial CC, to achieve a highly controllable hierarchical 3D architecture, we subsequently used optical emission spectroscopy (OES, Fig. S11 $\dagger$ ) for further analysis. As shown in Fig. S11, $\dagger$ there are three major regions in the emission spectrum of nitrogen plasma, representing different nitrogen species i.e. nitrogen in molecular, atomic and ionic forms. Among these, $\mathrm{N}_{2}{ }^{+}$molecular ions represented by emission peaks in the 350 to $450 \mathrm{~nm}$ range play an essential role in achieving the nitrogen doping as well as enhancing the roughness and nanostructured morphology of the treated CC surface (Fig. 5). $\mathrm{N}_{2}{ }^{+}$ions can deliver an excitation energy of $\sim 18.7 \mathrm{eV}$, leading to $\mathrm{CN}$ bonding with an activated state. ${ }^{29}$ The formation of a volatile $\mathrm{CN}$ phase during plasma activation is also thought to be responsible for the decrease in amorphous carbon for the treated CC and changes in the surface nanostructure. ${ }^{30}$ The etching effect of nitrogen plasma is relatively weak compared to that of $\mathrm{H}$ and $\mathrm{O}$ plasmas, so the graphitic architecture is relatively stable under $\mathrm{N}$ etching. ${ }^{31}$ Moreover, it has been reported that incorporated $\mathrm{N}$ in the $\mathrm{sp}^{3}$ configuration may function as a bridge between $\mathrm{sp}^{2}$ clusters, leading to localization of the $\pi$-and $\pi^{*}$-bands, which are responsible for aromatic ring clusters (graphitic micro-spherical domination). ${ }^{30}$ The $\mathrm{N}_{2}{ }^{+}$levels in the nitrogen plasma accelerate the formation of a graphitic microspherical assembly, leading to domination of the graphitic phase over the amorphous phase. Therefore, the hierarchical networks composed of aromatic ring clusters should survive and gradually accumulate with increasing plasma activation duration. It has been noted that the localized aromatic ring clusters also form and increase in number with varying several operational parameters, such as increased $\mathrm{N}_{2}$ flow rate, enhanced power density and elongated treatment duration. ${ }^{32}$

Based on the above analysis, the mechanism of the hNCC formation (shown in Fig. 5) can be summarized as follows:

(1) Before plasma activation, the untreated CC exhibits a smooth surface which is composed of amorphous and graphitic carbon;

(2) During nitrogen plasma treatment, the amorphous carbon reacts with and is etched by $\mathrm{N}_{2}{ }^{+}$ions to produce volatile $\mathrm{CN}_{x}^{+}$while the graphitic composition continuously accumulates to achieve a nano-architecture;

(3) Nitrogen-containing ions bond with graphitic carbon, particularly at the edges of the graphitic structure (based on XPS results);

(4) Nitrogen-doped graphitic micro-spherical particles are thus formed over the entire outer surface of the CC to achieve a hierarchical 3D nano-architecture.

\subsection{Effect of plasma power}

To further demonstrate the validity of the above explanation, we studied the effect of the plasma power on the surface morphology and the nitrogen content of the treated CC. The RF power was reduced to $250 \mathrm{~W}$ with the CC sample being treated for the same fixed duration of $15 \mathrm{~min}$. This sample is referred to as rNCC. Reducing the RF power significantly reduces the formation of a porous nanostructured surface on the CC (compare Fig. 6b for rNCC with Fig. 1b for hNCC) as only a randomly separated micro-spherical architecture is seen to form on the outer surface of the rNCC. Fig. 6a shows that for a reduced $\mathrm{RF}$ power of $250 \mathrm{~W}$, the intensity of the $\mathrm{N}_{2}{ }^{+}$emission peak and correspondingly the number of reactive $\mathrm{N}_{2}{ }^{+}$ions, is lower. This is further evidenced by the XPS results shown in Fig. $6 \mathrm{c}-\mathrm{d}$ and the data tabulated in Table $\mathrm{S} 1 . \dagger$ The overall nitrogen atomic percentage decreased from $5.6 \%$ for $500 \mathrm{~W}$ to $2.7 \%$ for $250 \mathrm{~W}$ RF plasma power. Notably, the percentage of $\mathrm{N}$ atoms on the edge of the graphite plane is also reduced to 88.1\% for rNCC (Table S1 $\dagger$ ), from $93.7 \%$ for hNCC. This investigation demonstrates that a higher abundance of the $\mathrm{N}_{2}^{+}$ level using a higher RF power is required not only to bond

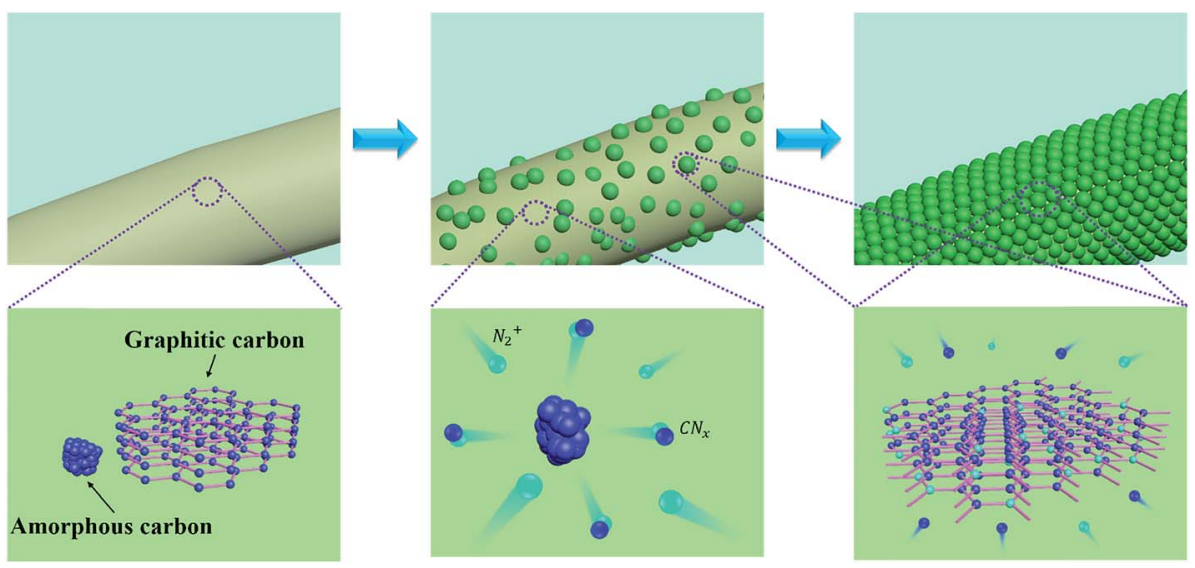

Fig. 5 A schematic formation process of the hNCC by plasma activation from the untreated CC. 

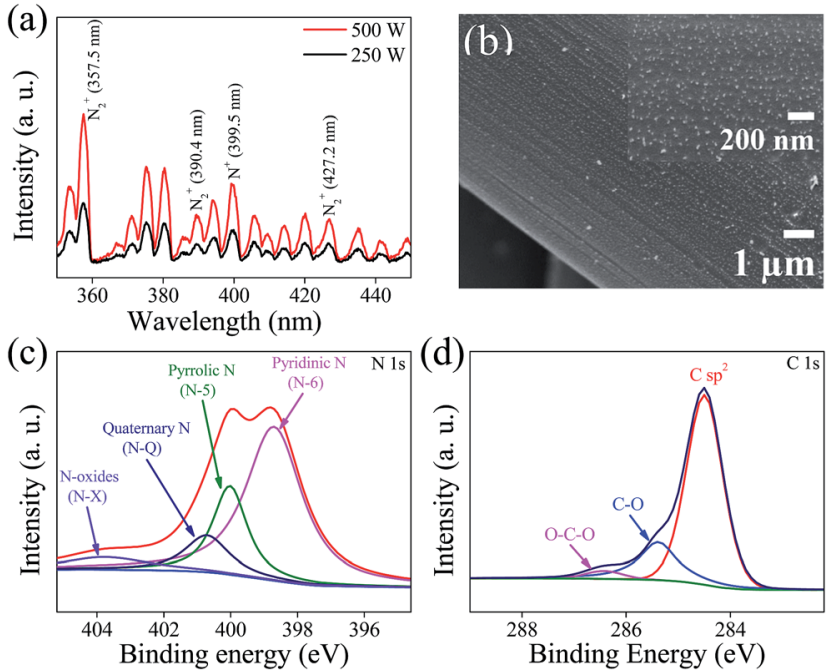

Fig. 6 (a) OES spectra of nitrogen plasma at different RF powers, 500 $\mathrm{W}$ (for the hNCC) and $250 \mathrm{~W}$ (for the rNCC sample); (b) SEM and FESEM images of the rNCC sample; (c) XPS spectra of N 1s in the rNCC; (d) XPS spectra of $\mathrm{C}$ 1s in the rNCC.

a significant amount of nitrogen with carbon atoms at the edge of the graphitic sheets but also to appropriately nanostructurize the treated carbon fiber surface.

Furthermore, we measured the supercapacitive performance of the rNCC to further confirm our results (data are shown in Fig. S12 $\dagger$ ). As shown in the resultant CV curve (Fig. S12a †) along with the GCD profile (Fig. S12b $\dagger$ ), the rNCC also delivered an irregular box-like shape as well as a capacitance of $237 \mathrm{mF} \mathrm{cm}^{-2}$ at $4 \mathrm{~mA} \mathrm{~cm}^{-2}$, which is much higher than that of the untreated CC but still lower than the hNCC. This also demonstrates that hNCC is capable of providing better performances due to its hierarchical nano-structure together with its enhanced nitrogen proportion.

\section{Conclusions}

In summary, we have demonstrated a facile, environmentallyfriendly, efficient and controllable approach based on RF nitrogen plasma processing of commercial carbon cloth to synthesize hierarchical nanostructured porous nitrogen doped CC as an excellent electrode material for energy storage devices. Upon plasma activation, the obtained hNCC exhibits a hierarchical nano-architecture composed of a graphitic nano-spherical porous assembly as well as abundant nitrogen-containing functional groups. Owing to these advantageous features, the as-prepared hNCC electrode delivers almost three times the Liion storage capacity compared to the untreated CC electrode, with excellent cyclic stability. Moreover, the hNCC has a specific capacitance of $391 \mathrm{mF} \mathrm{cm}^{-2}$ at a current density of $4 \mathrm{~mA} \mathrm{~cm}^{-2}$, three orders of magnitude higher than the value for the untreated CC electrode. Furthermore, a plausible mechanism to achieve such a hierarchical 3D architecture is investigated for not only confirming the achievement of an excellent performance in energy storage devices but also for providing a generalized approach to effectively control the morphology and nitrogen doping of carbon based materials by RF plasma treatment. We believe the plasma activation strategy to significantly enhance the capacitive performance of CC will open up a novel opportunity in the development of cost-effective and high-performance electrode materials for energy storage devices.

\section{Acknowledgements}

This study was supported by a NIE RS-SAA research grant RS 6/ 14 RSR provided by National Institute of Education, Nanyang Technological University, Singapore, and a Singapore MOE AcRF Tier 1 grant (RG98/15).

\section{Notes and references}

1 B. Luo and L. Zhi, Energy Environ. Sci., 2015, 8, 456.

2 D. Sun, J. Yang and X. Yan, Chem. Commun., 2015, 51(30), 6540 .

3 C. Wang, H. Chen, W. Dong, J. Ge, W. Lu, X. Wu, L. Guo and L. Chen, Chem. Commun., 2014, 50, 1202.

4 B. Ouyang, M. V. Jacob and R. S. Rawat, Mater. Res. Bull., 2015, 71, 61.

5 M. Yu, Y. Huang, C. Li, Y. Zeng, W. Wang, Y. Li, P. Fang, X. Lu and Y. Tong, Adv. Funct. Mater., 2015, 25, 324.

6 B. Liu, J. Zhang, X. Wang, G. Chen, D. Chen, C. Zhou and G. Shen, Nano Lett., 2012, 12, 3005.

7 X. Lu, T. Zhai, X. Zhang, Y. Shen, L. Yuan, B. Hu, L. Gong, J. Chen, Y. Gao, J. Zhou, Y. Tong and Z. L. Wang, Adv. Mater., 2012, 24, 938.

8 J. Xu, Q. Wang, X. Wang, Q. Xiang, B. Liang, D. Chen and G. Shen, ACS Nano, 2013, 7, 5453.

9 X. Lu, M. Yu, G. Wang, T. Zhai, S. Xie, Y. Ling, Y. Tong and Y. Li, Adv. Mater., 2013, 25, 267.

10 G. Zhang, S. Hou, H. Zhang, W. Zeng, F. Yan, C. C. Li and H. Duan, Adv. Mater., 2015, 27, 2400.

11 G. Wang, H. Wang, X. Lu, Y. Ling, M. Yu, T. Zhai, Y. Tong and Y. Li, Adv. Mater., 2014, 26, 2676; W. Wang, W. Liu, Y. Zeng, Y. Han, M. Yu, X. Lu and Y. Tong, Adv. Mater., 2015, 27, 3572 .

12 I.-Y. Jeon, M. J. Ju, J. Xu, H.-J. Choi, J.-M. Seo, M.-J. Kim, I. T. Choi, H. M. Kim, J. C. Kim, J.-J. Lee, H. K. Liu, H. K. Kim, S. Dou, L. Dai and J.-B. Baek, Adv. Funct. Mater., 2015, 25, 1170.

13 J. Zhao, H. Lai, Z. Lyu, Y. Jiang, K. Xie, X. Wang, Q. Wu, L. Yang, Z. Jin, Y. Ma, J. Liu and Z. Hu, Adv. Mater., 2015, 27, 3541.

14 J. Xu, Y. Lin, J. W. Connell and L. Dai, Small, 2015, 11, 6179. 15 L. Shen, J. Wang, G. Xu, H. Li, H. Dou and X. Zhang, Adv. Energy Mater., 2015, 5, 1400977.

16 Y.-H. Lee, K.-H. Chang and C.-C. Hu, J. Power Sources, 2013, 227, 300.

17 A. Ferrari, J. Meyer, V. Scardaci, C. Casiraghi, M. Lazzeri, F. Mauri, S. Piscanec, D. Jiang, K. Novoselov and S. Roth, Phys. Rev. Lett., 2006, 97, 187401.

18 A. C. Ferrari, Solid State Commun., 2007, 143, 47. 
19 K. T. Lee, X. Ji, M. Rault and L. F. Nazar, Angew. Chem., Int. Ed., 2009, 48, 5661.

20 H. Wang, T. Maiyalagan and X. Wang, ACS Catal., 2012, 2, 781.

21 X. Li, H. Wang, J. T. Robinson, H. Sanchez, G. Diankov and H. Dai, J. Am. Chem. Soc., 2009, 131, 15939.

22 T. Lin, I.-W. Chen, F. Liu, C. Yang, H. Bi, F. Xu and F. Huang, Science, 2015, 350, 1508.

23 J. Han, G. Xu, B. Ding, J. Pan, H. Dou and D. R. MacFarlane, J. Mater. Chem. A, 2014, 2, 5352.

24 R. H. Bradley, M. W. Smith, A. Andreu and M. Falco, Appl. Surf. Sci., 2011, 257, 2912.

25 W. Ai, Z. Du, Z. Fan, J. Jiang, Y. Wang, H. Zhang, L. Xie, W. Huang and T. Yu, Carbon, 2014, 76, 148.

26 C. Guan, X. Wang, Q. Zhang, Z. Fan, H. Zhang and H. J. Fan, Nano Lett., 2014, 14, 4852; C. Zhu, P. Yang, D. Chao, X. Wang,
X. Zhang, S. Chen, B. K. Tay, H. Huang, H. Zhang, W. Mai and H. J. Fan, Adv. Mater., 2015, 27, 4566.

27 P. Yang, P. Sun, L. Du, Z. Liang, W. Xie, X. Cai, L. Huang, S. Tan and W. Mai, J. Phys. Chem. C, 2015, 119, 16483.

28 N. Kuriyama, T. Sakai, H. Miyamura, I. Uehara, H. Ishikawa and T. Iwasaki, J. Alloys Compd., 1993, 202, 183.

29 A. Qayyum, S. Zeb, S. Ali, A. Waheed and M. Zakaullah, Plasma Chem. Plasma Process., 2005, 25, 551.

30 N. I. Klyui, Y. P. Piryatinskii and V. A. Semenovich, Mater. Lett., 1998, 35, 334.

31 C. A. Wolden, C. E. Draper, Z. Sitar and J. T. Prater, Diamond Relat. Mater., 1998, 7, 1178.

32 A. Voevodin, J. Jones, J. Zabinski and L. Hultman, J. Appl. Phys., 2002, 92, 724; G. Farrow and C. Jones, J. Adhes., 1994, 45, 29. 